\title{
Biochemical Indices of Cardaic Function of Albino Wistar Rats Exposed to Hippocratea Africana Root Bark Extract
}

\author{
Ndem, Jessie Idongesit ${ }^{1,}$ David, Imoh Kingsley ${ }^{2}$ \\ Department Of Biochemistry, Faculty Of Basic Medical Sciences, University Of Uyo, Uyo. Correspondence
}

\begin{abstract}
The effect of Hippocratea africana root bark extract used traditionally in the treatment of malaria in the South Eastern part of Nigerian on markers of cardiac function (creatine kinase-MB (CK-MB), Lactate dehydrogenase (LDH) and Troponin-I) on adult male albino Wistar rats was investigated. Twenty-four (24) adult male albino Wistar rats weighing between 100 - $160 \mathrm{~g}$ were used for the study. They were randomly divided into four (4) groups of six (6) animals each. Group I animals served as the control and were administered Iml distilled water. Groups II, III and IV served as the test groups and were administered 100, 200 and $300 \mathrm{mg} / \mathrm{kg}$ body weight of the root bark extract for 14 days, orally once daily by use of cannula attached to a syringe. All the experimental animals were given normal rat chow and water ad libitum throughout the treatment period. The result showed a dose-dependent significant $(p<0.05)$ decrease in serum CK-MB, LDH and troponin compared with the control. The deceases obtained inferred a non-cardiac function disturbance or derangement in critical tissues or organ function by the herb. The observed reductions in the concentration of markers of cardiac function imply that the herb maybe safe for its use for its antiplasmodial property.
\end{abstract}

Keywords - Cardiac function, Creatine kinase-MB, Hippocratea africana, Lactate dehydrogenase, Troponin.

\section{Introduction}

Malaria is a worldwide public health problem with enormous health and economic consequences. It is one of the major tropical parasitic diseases responsible for significant morbidity and mortality especially among children and pregnant women. It is estimated that one to two million people die yearly as a result of malaria [1]. World Health Organisation (WHO) stated that Nigeria accounts for a quarter of all malaria cases in Africa. In the southern part of Nigeria, transmission occurs all year round while in the north it is mostly seasonal [2].

Artemisinin Combination Therapy (ACT) is the current WHO recommended modality for malaria chemotherapy [3]. Even with its potency in combating the resistance falciparium strain of malaria parasite, some factors have been reported to limit ACT's use such as its high cost, limited production of artemisinin-derivatives to Good Manufacturing Practices (GMP) Standards, toxicity and others [4][5][6].

Recently (in the 2000s decade), malaria with partial resistance to artemisinins has been reported in Southeast Asia [7][8]. In view of the problems associated with antimalarial drug resistance, new drugs or drug combinations are urgently required for treatment of malaria. Preferably, the new drugs should have novel modes of action or be chemically different from the drugs in current use [9]. Plants have always been considered to be a possible alternative and rich source of new drugs and most of the antimalarial drugs in use today such as quinine and artemisinin were either obtained directly from plants or developed using chemical structures of plantderived compounds as templates [10]. Herbal usage seems necessary due to the resistance of the malaria parasite to conventional antimalarial drugs and persistence of malaria symptoms after treatment with these antimalarial drugs.

In poor countries, the healthcare is often sustained by other practices based on cultural alternatives. Data available in many developing countries show that one-fifth of patients use indigenous herbal remedies to treat malaria [11]. It has been observed by Adebayo and Krettli (2011) that some of these herbal remedies are used in combination with other medicines. Also, there are reports of a renewed interest in indigenous medicine worldwide in the last decade, arising from the realization of the limitations of orthodox drugs [2].

Hippocratea africana (Hippocrateaceae) is a green forest glaborous perennial climber that reproduces from seeds [12]. The root bark of the plant is used traditionally in the treatment of various ailments such as malaria, fever, body pains, diabetes, and diarrhea [13]. Its antimalarial property has been reported in mice [13] and there are many reports on some of its effects on biochemical parameters [14][15][16][17]. The root bark extract of the herb is used traditionally by people in the South Eastern part of Nigeria for the treatment of malaria. Damage to the heart muscle by toxin is called cardiac toxicity. There has been increasing report of ischaemic heart disease which may be attributed to an increase in the prevalence of known cardiovascular risk factor including drugs and local herbs [18][15]. Cardio toxicity and its related diseases have reported following drugs used in the treatment of malaria [19][20][21]. Cardiovascular disease (CVD) has been described as the major cause of morbidity and mortality in adult humans [18]. Epidemiological studies have shown that elevated concentrations of serum total cholesterol and LDL-cholesterol, triglycerides, fibrinogen and platelet count are 
independent risk factors for CVD [19]. Verlinde et al., (2016) [20] reports that prolonged administration of chloroquine can cause heart block and progressive myopathy. Ikezoe et al., (2009) [22] reports that chloroquine triggers the disruption of lysosomal enzymes, including amyloid- $\beta$ accumulation and endoplasmic reticulum (ER) stress. Amyloid- $\beta$ causes skeletal muscle fibre degeneration, activating autophagy in skeletal muscles of rats. Cases of neuromyopathies and cardiomyopathies due to chronic chloroquine intoxication have also been reported in long term therapies of autoimmune disorders (mainly in systemic lupus erythematous) and during the use of chloroquine in malaria prophylaxis [21]. According to reports by Silamut et al., 1995[23], quinine can cause serious toxic effects such as arrhythmia, angina, hypotension, circulatory defect and shock. Case reports of severe malaria patients presenting arrhythmia during a treatment with quinine have been documented [19]. A fatal ventricular fibrillation has also been reported in a malaria patient, with no heart disease history, probably due to the toxicity of the administered quinine during the treatment [24].

Artesunate has been used as an alternative to treat malaria with minimal toxicity. However, it has been shown to affect airway smooth muscles, inhibiting the proliferation of human cultured airway smooth muscle cells, causing hyperplasia and hypertrophy leading to obstruction of airways [25]. In a report by Hitti, (2004) [26], administration of high doses of artesunate was shown to have cardio toxic effect. There is a consistent cardio toxicity reports on large scale use of Halofantrine, a phenatrane-methanol that is highly effective for $P$. falciparum malaria [27]. Bassi et al., (2006) [28], reported significantly increase activities of liver transaminase (ALT and AST) as well as cardiac enzymes (CK and LDH) following administration of halofantrine compared to baseline values. Damage to any of these tissues (cardiac and skeletal muscles, liver, kidney and erythrocytes) due to toxic injury such as drugs may increase plasma AST levels. Significant increase in the serum level (10 100 folds) of AST indicates severe damage to liver (viral hepatitis or toxic liver necrosis) or heart muscles (myocardial infarction) [29]. There is no literature of Hippocratea africana root bark on cardiac enzymes. This paucity of information on indices of cardiac function necessitated this study on the effect of Hipporeatea africana root bark extract on creatine kinase (CK-MB), lactate dehydrogenase (LDH) and troponin (cTn-1).

\subsection{Plant Sample}

\section{Materials and Methods}

The plant part (root) was collected from Afaha Etok forest in Ibesikpo- Asutan Local Government Area, Akwa Ibom State. It was identified and authenticated by a taxonomist in the Department of Botany and Ecological Studies, University of Uyo. Uyo. The voucher number (UUH3394) was given.

The roots were gently washed to get rid of debris. The root bark was scrapped, cut into small pieces and pulverized. The pulverized sample was macerated in $80 \%$ ethanol (Sigma Aldrich) for 72 hours. Within this period, the mixture was shaken thrice in every 24 hours to allow the solvent to solubilize the active phytochemicals. After 72 hours, the clear orange colour supernatant was carefully siphoned off and concentrated to dryness in a water bath at $45^{\circ} \mathrm{C}$ to obtain the crude extract.

\subsection{Experimental Animals}

Twenty-four (24) mature male albino Wistar rats weighing between 110-150g used in this study were obtained from the Animal House, Department of Pharmacology and Toxicology, Faculty of Pharmacy, University of Uyo. The animals were divided into four groups of six (6) rats per group. They were housed in a ventilated room in standard laboratory cages under standard laboratory conditions and allowed to acclimatize for 7 days before the study. The animals were fed grower's mash (guinea feed) and water were provided ad libitum throughout the experimental period (14 days). Group I was the control and was administered $1 \mathrm{ml}$ distilled water, Groups II, III and IV served as the test groups and were administered 100, 200 and $300 \mathrm{mg} / \mathrm{kg}$ body weight of the root bark extract respectively, orally once daily by use of cannula attached to a syringe.

\subsection{Blood Collection and Preparation of Sera}

The animals were denied food for 24 hours after the administration of the last dosage of the extract but were still allowed water ad libitum. They were chloroform anaesthetized and blood samples were obtained by cardiac puncture using sterile needles and syringes into sterile plain tubes. Serum was obtained by centrifugation of the clotted blood at $4000 \mathrm{rpm}$ for 10 minutes using a bench top centrifuge.

\subsection{Biochemical Assays}

Serum activities of Creatinine Kinase (CKM), Lactate Dehydrogenase (LDH) and Cardiac Troponin-I were estimated using laboratory kits and methods described by Kamiya Biomedical Company, 12279, Gateway Drive, Seattle, WA98168. The absorbance was read using micro-plate reader. 
2.5Statistical Analysis

All the results were presented as mean \pm standard deviation (SD). One-way analysis of variance (ANOVA) was employed for comparison to assess statistical significance using widows SPSS. Probability level $<0.05$ was considered significant.

\section{Results}

The effect of administration of Hippocratea africana root bark extract (100, 200 and 300mg/kg body wt.) to male albino Wistar on some biochemical markers of cardiac function is presented in the Figures 1 and 2 below. The result showed a dose-dependent decrease in cardiac enzymes (CK-MB, LDH and Troponin-I).
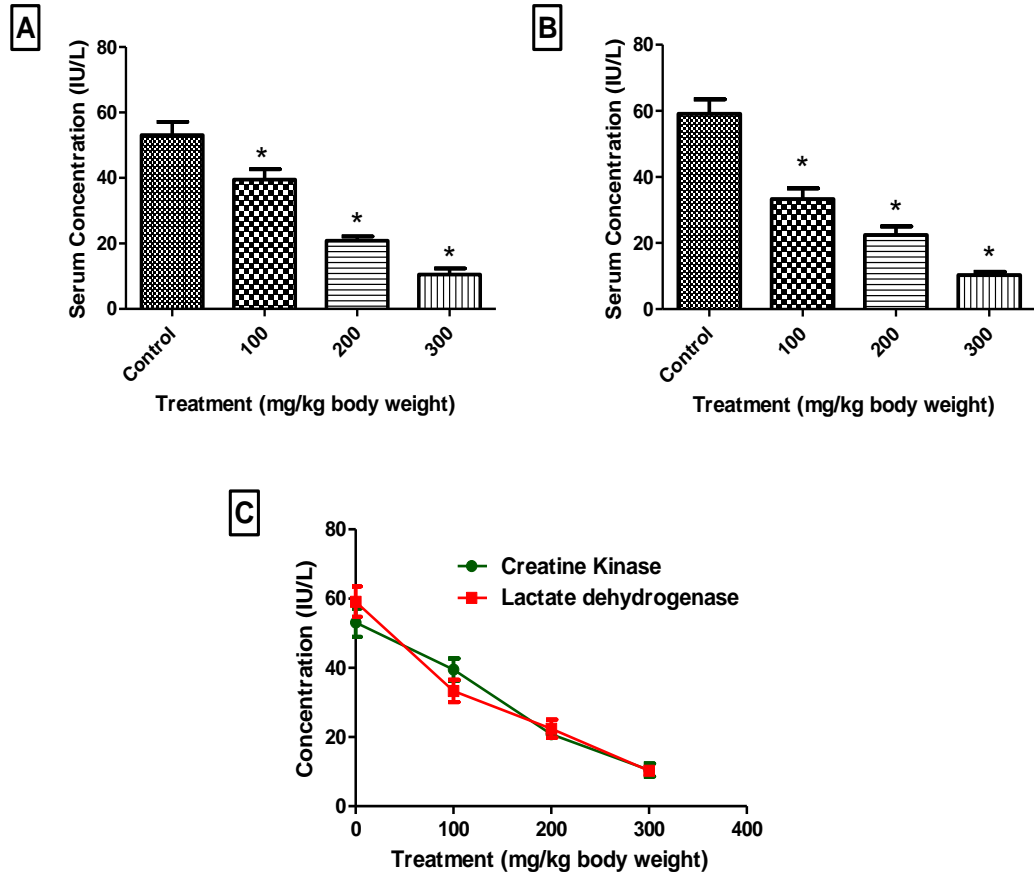

Figure 1: Showing Serum concentrations of Creatine Kinase (A); Lactate dehydrogenase (B) and Line graph (C) showing A and B together.

$*=$ Test treatments statistically significant $(P<0.05)$ compared with the control.
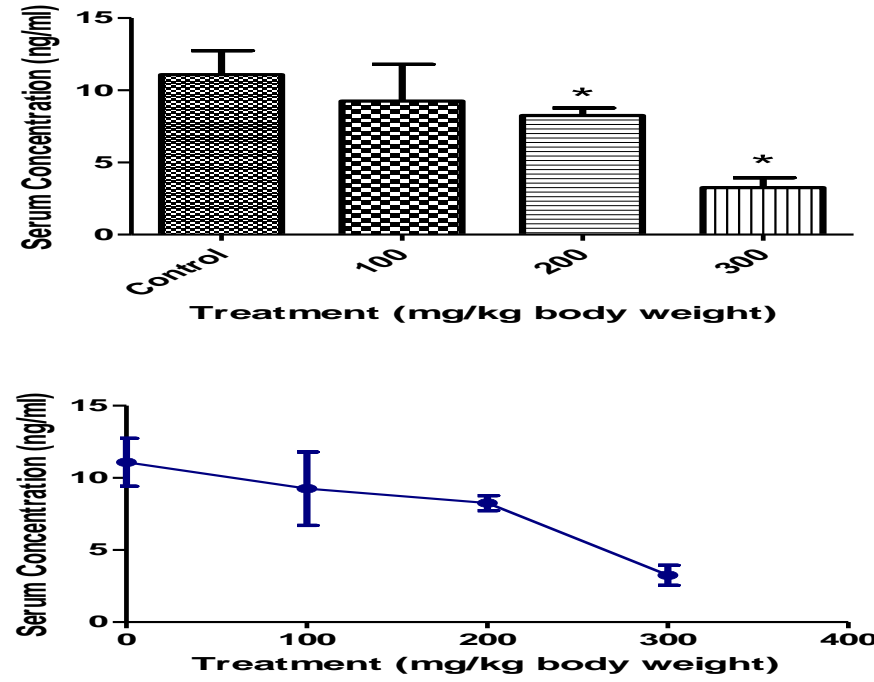

Fig. 2: Serum concentration of Troponin.

$*=$ Test treatments statistically significant $(P<0.05)$ compared with the control. 


\section{Discussion}

Malaria remains one of the most prevalent infectious diseases in the world. It is a significant public health problem associated with poverty and is one of the main obstacles to the economy of an endemic country. Recently, challenges of malaria treatments emanate from resistant strains of malaria parasite to the unavailability and unaffordability of the Artemisinin Combination Therapy (ACT) recommended by the World Health Organization (WHO). This has necessitated a fall-back to herbal remedies for the treatment of malaria as well as other ailments. Herbal medicines have been decisive in the treatment of some diseases; however, in the face of the increasing use and fast-growing market of herbal medicines, health professionals and the public are increasingly expressing concerns about the safety, efficacy, quality and preservation of these herbal drugs. Thus, the evaluation of anti-malarial herbal agents for possible cardio toxic or cardio protective effect is important.

This study reports the effect of ethanol root bark extract of Hippocratea africana on cardiac enzymes. The present study revealed that administration of $H$. africana to adult male albino Wistar rats caused significant $\mathrm{p}<0.05$ decrease in the cardiac markers (CK-MB, LDH and Troponin). The significant $\mathrm{p}<0.05$ decrease may be due to the phytochemicals such as cardiac glycosides and flavonoids present in the herb [15]. Cardiac glycosides have been reported to have an inverse relationship with cardiovascular disease [30]. Flavonoids have been reported to possess vasodilatory as well as inhibitory effects on platelets, coronary heart disease [15][31].

Creatine kinase $(\mathrm{CK})$ is an enzyme present in many parts of the body (muscles, brain, colon and urinary bladder). Its physiological role is to maintain an adequate store of high energy phosphorylated creatine used to restore ATP levels depleted during muscle contraction. CK is composed of two subunits existing in three molecular forms namely- CK-MM, CK-MB and CK-BB. CK-MB predominates in the heart muscle and CK$\mathrm{MM}$ is most prevalent in the skeletal muscle. It is found in the mitochondria and cytoplasm of skeletal muscle, cardiac muscle, brain and other visceral tissues. Its main function is in the transportation of high energy phosphate group. Serum total CK activity and CK-MB concentration rise in parallel following myocardial injury. A significant elevation in the level of CK-MB has been observed in the heart effluent during myocardial ischemia band reperfusion in isolated rat hearts [32]. Jaffe et al., (2000) also reported that serum CK-MB is considerably more specific for myocardial damage [33].

In this study, there was an observed dose-dependent reduction in the concentration of CK following administration of $H$. africana to Wistar rats. Increased $\mathrm{CK}$ is a marker for neuromuscular diseases and acute myocardial infarction. Neuromuscular disorders include myopathies, muscular dystrophy, rhabdomyolysis, drug-induced myopathy, neuroleptic malignant syndrome, malignant hyperthermia and periodic paralyses which result in cardio toxicity. The dose-dependent decrease observed this study suggests that the herb did not induce any neuromuscular disorder and myocardial infarction. This effect may be further attributed to the flavonoids and cardiac glycosides richly present in the herb.

Lactate dehydrogenase (LDH) also recorded significant dose dependent decrease compared with the control. LDH is an enzyme present in all cells of the body with highest concentrations in the heart, liver, muscles, kidney, lungs and erythrocytes. It exists in a tetrameric isoform and can form five possible tetramers of which LDH-1 (4H) is the most often used indicator of heart pathology [34]. In normal conditions, LDH-2 concentration is greater than LDH-1, but in myocardial infarction, there is a "flip" in the ratio resulting in LDH1: LDH-2 greater than 1.

The observed significant decrease in LDH may be due to the phytochemicals flavonoids and cardiac glycosides richly present in the herb [15]. Cardiac glycosides have been reported to be cardioactive compounds with their inherent property on the aglycone portion of the sugar moiety that exert a number of efforts on neural tissue thereby indirectly influencing the mechanical and electrical activities of the heart, modifying vascular resistance and capacitance [15][35]. An elevated level of LDH-1 is a marker for blood flow deficiency, cerebrovascular accident (stroke), heart attack, muscle injury and muscular dystrophy. The dose-dependent decrease observed suggest that the herb did not induce cardiovascular injury.

Cardiac troponin was observed to significantly reduce following administration of ethanol extract of $H$. africana root bark in a dose dependent manner. Cardiac troponins (cTn), an inhibitory protein complex in all striated muscles consists of three subunits (I, T and C). Troponins do not exist in the blood of healthy individuals or are suggested to exist in very small amounts [36]. The cardiac troponins (cTnI and cTnT) are the "gold standard" for myocardial injury due to their great sensitivity, specificity and better efficacy [37]. Cardiac troponins have been highlighted by their roles in cardio specific diagnosis, prognostic risk assessment, detection of myocardial re-infarction, detection of coronary reperfusion and renal failure [38][39].

An elevated cTn levels indicate cardiac injury including acute perimyocarditis, acute coronary injury including acute pulmonary embolism, acute heart failure and tachycardia [40]. The dose-dependent decrease observed suggests that the herb did not induce any cardiac injury. This effect may be attributed to the flavonoids and tannins present in the herb. Tannins have been reported to inhibit oxidation of LDL-cholesterol, reduce body fat and thereby decrease incidence of heart disease [41]. The dose- dependent decrease in the concentration of troponin observed in this study may also indicate that the herb did not cause any oxidative stress in the 
experimental animals. Ndem and Ewere, (2015) reported significant increases in AST concentration following administration of the herb to male rats that was attributable to the protective effect of the antioxidant flavonoid [16]. This further shows that the herb did not induce oxidative stress and may be cardio protective.

\section{Conclusion}

In this study, there was significant reduction in the biochemical markers of cardiac function as seen in the decreased concentration of CK-MB, LDH and Troponin-1. The biochemical indices taken together do not suggest cardiac malfunction or derangement in critical tissues or organ function. Hence, the herb may be safe for use for its antiplasmodial property.

\section{References}

[1]. W. H. O. Global Malaria Programme. World Malaria Report. 2014. http://www.who.int/malaria/publications/world-malariareport/en/

[2]. JO Adebayo and AU Krettli. Potentials of Antimalarials from Nigerian Plants: A Review. Journal of Ethnopharmacology, 133, 2011, 289-302

[3]. NJ White. Antimalarial Drug Resistance and Combination Chemotherapy. Philosophical Transactions of the Royal Society of London, 354(6), 1999, 739-749.

[4]. RK Haynes. Artemisinin and Derivatives: The Future for Malaria Treatment? Current Opinion in Infectious Diseases, 2001, 14, 719-726.

[5]. AC Boareto, JC Muller, AC Bufalo, GK Botelho, SL de Araujo, MA Foglio, RN de Morais and PR Dalsenter. Toxicity of Artemisinin in two Different Periods of Pregnancy in Wistar rats. Reproductive Toxicology, 25, 2008, $239-246$.

[6]. JO Adebayo and SO Malomo. The Effect of Co-administration of Dihydroartemisinin with Vitamin E on the Activities of Cation ATPases in some Rat Tissues. Nigerian Journal of Pure and Applied Sciences, 17, 2002, 1245-1252.

[7]. C O'Brien, PP Henrich, N Passi and DA Fidock. Recent Clinical and Molecular Insights into Emerging Artemisinin Resistance in Plasmodium falciparum. Current Opinion in Infectious Diseases, 24(6), 2011, 570-577.

[8]. RM Fairhurst, GM Nayyar, JG Breman, R Hallet, JL Vennerstrom, S Duong, P Ringwald, TE Wellems, CV Plowe and AM Dondrop. Artemisinin-Resistant Malaria: Research Challenges, Opportunities, and Public Health Implications. American Journal of Tropical Medicine and Hygiene, 87(2), 2012, 231-241.

[9]. JD Phillipson and CW Wright. Antiprotozoal Agents from Plant Sources. Planta Medica, 57(1), $1991,53-59$.

[10]. HA Ibrahim, IA Imana, AM Bellob, U Umara, S Muhammada and SA Abdullahi. The Potential of Nigerian Medicinal Plants as Antimalarial Agents. A Review of International Journal of Science and Technology, 2, 2012, 8.

[11]. ML Willcox and G Bodeker. Traditional Herbal Medicine for Malaria. BMJ 329(7475), 2004, 1156-1159.

[12]. JM Dalziel. Useful Plants of West Tropical Africa. Crown Agents for Overseas Government (London. 1956) $403-404$.

[13]. JE Okokon, BN Ita and AE Udokpoh. The In Vivo Antimalarial Activities of Uvariae chamae and Hippocratea africana. Annals of Tropical Medicine and Parasitology, 100(9), 2006, 585-590.

[14]. JI Ndem, MU Eteng, MI Akpanabiatu, AF Uwah, O Otitoju and EO Akpanyung. Effect of Hippocratea africana Root Bark Extract on some Biochemical Indices of Male and Female Albino Wistar Rats. Journal of Pharmacognosy and Phytotherapy, 5(4), 2013, $72-76$.

[15]. JI Ndem, MU Eteng and AF Uwah. Effect of Hippocratea africana Root Bark Extract on Lipid Profile of Female and Male Albino Wistar Rats. Journal of Scientific Research and Reports, 3(19), 2014, 2574-2583.

[16]. JI Ndem and EG Ewere. Comparative Hepatic Effect of Hippocratea africana Root Bark Extract on Female and Male Albino Wistar Rats. British Journal of Pharmaceutical Research, 9(3), 2015, 1-11.

[17]. JI Ndem, E Uka, AF Uwah and AI Peter. Renal Status of Female and Male Wistar Rats Exposed to Hippocratea africana Root Bark Extract. European Journal of Pharmaceutical and Medical Research, 3(3), 2016, 82-88.

[18]. JB John, AC Patricia, DC Anthony and RM Macknight. Cardiovascular System in Lecture Notes in Physiology. 4th edn. (Blackwell Science Ltd., UK. 1999) 344-346.

[19]. CA Gunawan, PN Harijanto, A Nugroho. Quinine-induced arrhythmia in a patient with severe malaria. Acta Med Indonesia. 39, 2007, 27-32.

[20]. BK Verlinde, A Louw and LM Birkholtz. Resting Resistance: Is there a Solution for Malaria? Expert Opinion on Drug Discovery, 29(2), 2016, 1-12.

[21]. F Lhermitte, R Marteau, F Chedru, J Mallecourt, G Estrade, J Godet-Gullain and M Chevallay. Chloroquine in Neuromyopathy. One Case of Prophylactic Malaria Therapy. Nouv Presse Med, 6, 1977, 3205-3207.

[22]. K Ikezoe, H Furuya, H Arahata, M Nakaga, T Tateshi and N Fujii. Amyloid-beta Accumulation caused by Chloroquine Injections Precedes ER Stress and Auto Phagosome Formation in Rat Skeletal Muscle. Acta Neuropathology, 117, $2009,575-582$.

[23]. K Silamut, R Hough, T Eggelte, B Angus and NJ White. A Simple Method for Assessing Quinine Pre-treatment in Acute Malaria. Transactions of the Royal Society of Tropical Medicine and Hygiene, 89, 1995, $665-667$.

[24]. A Bonington, RN Davidson, PA Winstanley and G Pasvol. Fatal Quinine Cardiotoxicity in the Treatment of Falciparum Malaria. Transactions of the Royal Society of Tropical Medicine and Hygiene, 90, 1996, 305-307.

[25]. C Cheng, WE Ho, FY Goh, SP Guan, LB Kong, WQ Lai, BP Leung and WSF Wong. Anti-Malarial Drug Artesunate Attenuates Experimental Allergic Asthma Via Inhibition of the Phosphoinositide 3-kinase/Akt Pathway. PloS ONE 6(6), 2011 , e20932.

[26]. M Hitti. Heart Disease Kills every 34 Seconds in US. 2004. http://www.foxnews.com/story/0,2933,142436,00.html.

[27]. NB Molta. Susceptibility of Plasmodium falciparium to Malarial Drugs in North-Eastern Nigeria. Trans. Roy. Trop. Med. Hyg. 89, $1995,422-425$.

[28]. PU Bassi, BI Buratai and W Kuchali. Effect of Halofantrine Administration on some Liver and Heart Enzymes in Healthy Human and Volunteers. African Journal of Biomedical Science, 9(1), 2006, 31-36.

[29]. H Thiagarajan, U Thiagamoorthy, K Gunasekaran, S Palavesam and P Rengarajulu. Enzymes in Clinical Medicine: An Overview. Indian Journal of Experimental Biology, 51, 2013, 777-788.

[30]. C Manach, A Mazur and A Scalbert. Polyphenols and Prevention of Cardiovascular Disease. Current Opinion in Lipidology, 6(1), 2005, 77-84.

[31]. S Suberamani and CA Casimr. Flavonoids and Anti-Oxidant Activity of Geogia Grown Vidalia Onions. Journal of Agricultural and Food Chemistry, 50(19), 2002, 5338-5342. 
[32]. M Tiwari, T Hemalatha, K Ganesan, BM Manohar, C Balachandra, S Vairamuthu, S Subramaniam and R Puvanakrishnan. Myocardial Ischemia and Reperfusion Injury in Rats: Lysosomal Hydrolases and Matrix Metalloproteinases Mediated Cellular Damage. Molecular and Cellular Biochemistry, 312, 2008, 81.

[33]. AS Jaffe, J Ravkilde, and R Roberts. It's Time for a Change to a Troponin Standard. Circulation, 102, 2000, 1216-1220.

[34]. Tietz. Textbook of Clinical Chemistry and Molecular Diagnostics: Burtis, CA. and Ashwood ER. (eds). (Philadelphia, PA: W.B Saunders Company, 2006).

[35]. SY Dangi, CI Jolly and S Narayana. Antihypertensive Activity of the Total Alkaloids from the Leaves of Moringa oleifera. Pharmaceutical Biology, 40(2), 2002, 144-148.

[36]. J Archer. Cardiac Troponin I: Evaluation of a Biomarker of the Diagnosis of Heart Disease in Dog. Journal of Small Animal Practice, 46, 2005, 139-145.

[37]. K Babuin and AS Jaffe. Troponin: The Biomarker of Choice for the Detection of Myocardial Injury. Canadian Medical Association Journal, 173, 2005, 1191-1202.

[38]. RP Jagnnadha, MJ Abdalla, H Abdul, KR Avinaash, DK Shoba, S Shakila, K Lokesh and R Narsinga. Cardiac Biomarkers: The Troponins and CK-MB. Ibnosina Journal of Medicine and Biomedical Sciences, 2(5), 2010, 190-197.

[39]. MW Scott and S Meg. Cardiac Troponins. Journal of Veterinary Emergency and Critical Care, 18(3), 2008, 235-245.

[40]. DM Vasudevan, S Sreekumari and V Kannnan. Textbook of Biochemistry for Medical Students, Seventh edition. (Jaypee Brothers Medical Publishers, New Delhi, 2013) 301-310.

[41]. Y Ishikawa, JE Saffilz, TL Meatmah, AM Grace. and R Roberts. Cardiac Biomarkers. Clinical Chemistry, 43, 1997, 467-475. 